\title{
Combination Therapy of Polymyxin B and Amikacin for Community-Acquired Pseudomonas aeruginosa Pneumonia with MODS in a Previously Healthy Patient: A Case Report
}

\author{
Junyu Wang' \\ Lu Yun' \\ Hong Zhao' \\ Xiangpeng $\mathrm{Li}^{2}$ \\ 'Department of Pharmacy, Qingdao \\ Central Hospital, Qingdao, 266042, \\ Shandong, People's Republic of China; \\ ${ }^{2}$ Department of Pharmacy, The Affiliated \\ Hospital of Qingdao University, Qingdao, \\ 266003, Shandong, People's Republic of \\ China
}

Background: Pseudomonas aeruginosa (P. aeruginosa) is an uncommon but fatal causative pathogen for community-acquired pneumonia (CAP). Few case reports described the previously healthy individuals with CAP caused by $P$. aeruginosa.

Case Presentation: A 67-year-old male farmer was referred to our hospital with a 6-day history of fever and shortness of breath. The radiologic findings revealed a dense consolidation site in the right lung, considering infection lesion, accompanying with mediastinal and right hilar lymphadenopathy. Sputum sample results showed $P$. aeruginosa and multidrugresistant Acinetobacter baumannii. The combination of polymyxin B and amikacin was given to him based on the lab results, the manifestations of the patient were alleviated and he returned to his home after a 35-day hospitalization.

Conclusion: We need to keep close attention to community-acquired $P$. aeruginosa pneumonia due to its high mortality, further studies are needed to identify the infectious source, effective examination, and optimal therapy including medications and duration.

Keywords: Pseudomonas aeruginosa, community-acquired pneumonia, polymyxin B and amikacin, a case report

\section{Background}

Pseudomonas aeruginosa pneumonia is caused by $P$. aeruginosa infection, which is a gram-negative aerobic bacterium heading the list of pathogens associated with hospital-acquired pneumonia. $P$. aeruginosa is the conditioned or opportunistic pathogen, widely distributing in the humid natural environment and body surface. $P$. aeruginosa pneumonia is usually seen in patients with lung structural change, immunocompromised, or other specific risk factors. ${ }^{1}$ Generally, although most of the patients are non-immunocompromised, the clinical course of community-acquired pneumonia (CAP) is considered to be more acute and severe. ${ }^{2}$ Recently years, the cases reported of previously healthy individuals with CAP caused by $P$. aeruginosa gradually increase, ${ }^{3-5}$ and the close relationship between mortality and $P$. aeruginosa pneumonia-related multiple organ dysfunction syndrome (MODS) have sparked widespread enthusiasm. Here, we describe a previously healthy 67-year-old male patient who developed severe $P$. aeruginosa CAP, and provide a literature review of $P$. aeruginosa CAP in terms of epidemiology, risk factors, pathogenesis and treatment.
Correspondence: Xiangpeng Li Department of Pharmacy, The Affiliated Hospital of Qingdao University, 16 Jiangsu Road, Shinan District, Qingdao, 266003, Shandong, People's Republic of China Tel +86-532-82912182; +86-18661809083 Email XiangpengLi021966@I63.com 


\section{Case Presentation}

A 67-year-old male with a 6-day history of fever and shortness of breath was admitted to the emergency department on August 15, 2019. This patient had a fever, chill, cough, and progressive dyspnea after being in the rain on August 9, 2019, without a sore throat, chest pain, hemoptysis, paroxysmal dyspnea, dizziness, nausea, abdominal distension, abdominal pain or diarrhea. He first went to a local clinic and was treated with "anti-inflammatory drugs (the specific name is unknown)", but his symptoms became more severe and rapidly progressive. One day before admission, the patient started having aggravated dyspnea, listlessness, and difficulty talking while lying down, then this patient presented to our emergency room (ER).

The physical exam revealed the following: $\mathrm{T} 36^{\circ} \mathrm{C}$, P 82bpm, RR 28/min, BP $107 / 64 \mathrm{mmHg}, \mathrm{SpO} 272 \%$, WBC $20.36 \times 10^{\wedge} 9 / \mathrm{L}, \mathrm{N} 18.31 \times 10^{\wedge} 9 / \mathrm{L}$; arterial blood gas (ABG) showed $\mathrm{PH} 7.12, \mathrm{PO}_{2} 55 \mathrm{mmHg}, \mathrm{PCO}_{2} 56 \mathrm{mmHg}$, $\mathrm{SaO}_{2} 85 \%$, Lactate $4.4 \mathrm{mmol} / \mathrm{L}, \mathrm{BE}-11.7 \mathrm{mmol} / \mathrm{L} ; \mathrm{BUN}$ $18.1 \mathrm{mmol} / \mathrm{L}$, creatinine $166 \mathrm{umol} / \mathrm{L}$; BNP $249.83 \mathrm{pg} / \mathrm{mL}$ with peripheral cyanosis and bilateral wheezing. Chest computed tomography (CT) showed a dense consolidation site in the right lung with scattered shadows in both lungs, accompanying by mediastinal and right hilar lymphadenopathy, the CT results indicated the infection lesion (Figure 1). He had been healthy previously, but he was a smoker with a 30-year history. He did not use illicit drugs and had no known allergies to medications. He worked as a farmer and have no family history. He was diagnosed as follows: 1 . Severe pneumonia 2. Sepsis 3. Septic shock 4. Multiple organ dysfunction syndrome (MODS) 5. Type II respiratory failure 6. Heart failure 7. Acute renal insufficiency 8. Metabolic acidosis 9. Chronic bronchitis.

Laboratory examinations were supplemented and the results were as follows (August 31, 2019): Blood routine: neutrophil count $7.29 \times 10^{\wedge} 9 / \mathrm{L}$, lymphocyte count $0.97 \times 10^{\wedge} 9$ / L, monocyte count $0.82 \times 10^{\wedge} 9 / \mathrm{L}$, neutrophil percentage $79.2 \%$, lymphocyte percentage $10.5 \%$, red blood cell count $3.01 \times 10^{\wedge} 12 / \mathrm{L}$, hemoglobin $86 \mathrm{~g} / \mathrm{L}$, hematocrit $0.28 \mathrm{~L} / \mathrm{L}$, mean corpuscular hemoglobin $305 \mathrm{~g} / \mathrm{L}$, platelet distribution width 9.2fl, C-reactive protein (CRP) $106.44 \mathrm{mg} / \mathrm{L}$; hepatorenal function: ALT 15U/L, AST 13U/L, Creatinine 75umol/L. Orotracheal intubation was applied to help the patient's respiration, assisting with the PB840 ventilator. Combined with the examination results of chest $\mathrm{CT}$, the patient was provided with imipenem and cilastatin sodium $1.0 \mathrm{~g} \mathrm{q} 12 \mathrm{~h}$, linezolid $0.6 \mathrm{~g} \mathrm{q} 12 \mathrm{~h}$, and moxifloxacin $0.4 \mathrm{~g}$ qd for antiinfection therapy. After a 3-day treatment, this patient still had a high fever with a maximum body temperature of $38.5^{\circ} \mathrm{C}$. Continuous invasive mechanical ventilation was performed to assist the respiration, meanwhile, continuous vasoactive drugs and intravenous nutrition support were given to him.

Sputum cultures were collected and Gram stain revealed no squamous epithelial cells, few neutrophils, and Gram-negative rods. Within one day, sputum sample results showed $P$. aeruginosa and multidrug-resistant Acinetobacter baumannii (Table 1). Besides, fiberscope secretion cultures showed $P$. aeruginosa, which is sensitive to amikacin, gentamicin and piperacillin sodium and tazobactam sodium, intermediate to ciprofloxacin and ceftazidime, but resistant to imipenem and meropenem (Table 2). Based on the culture results, we changed the treatment strategy to the combination of polymyxin B (50IU, q12h) and amikacin $(0.6 \mathrm{~g}, \mathrm{qd})$ for 30 days, instead of imipenem (1g q12h) and cilastatin sodium (500mg, q12h).

The patient's body temperature was back to $37^{\circ} \mathrm{C}$, measured by a clinical thermometer for armpit use for 10 minutes. His cough was significantly alleviated with white viscous sputum. On day 21, laboratory examinations
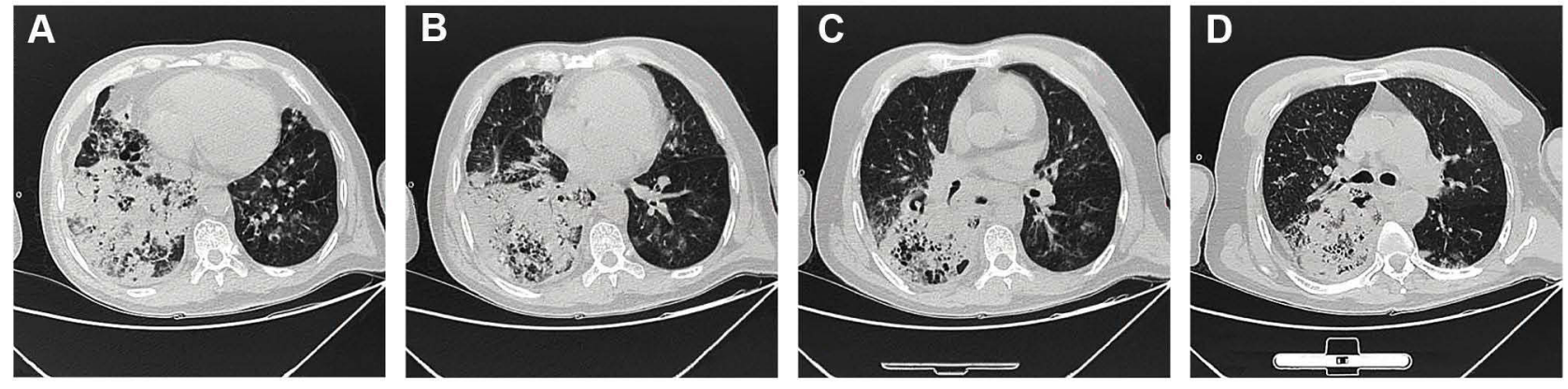

Figure I Computerized tomography was performed when the patient was admitted to the hospital. CT showed a dense consolidation site in the right lung, accompanying by mediastinal and right hilar lymphadenopathy. 
Table I Drug Sensitivity Test of A. baumannii

\begin{tabular}{|c|c|c|}
\hline \multicolumn{3}{|l|}{ A. baumannii ++++ } \\
\hline Antibiotics & MIC (ug/mL) & Results \\
\hline Amikacin & $>32$ & Resistance \\
\hline Ampicillin/Sulbactam & $>16 / 8$ & Resistance \\
\hline Cefepime & $>16$ & Resistance \\
\hline Cefotaxima & $>32$ & \\
\hline Ceftazidime & $>16$ & Resistance \\
\hline Ciprofloxacin & $>2$ & Resistance \\
\hline Polymyxin & $>8$ & \\
\hline Gentamicin & $>8$ & Resistance \\
\hline Imipenem & $>8$ & Resistance \\
\hline Levofloxacin & $>8$ & Resistance \\
\hline Meropenem & $>8$ & Resistance \\
\hline Piperacillin & $>64$ & Resistance \\
\hline Piperacillin/ Tazobactam & $>64 / 4$ & Resistance \\
\hline Tetracycline & $>8$ & Resistance \\
\hline Cotrimoxazol & $>2 / 38$ & Resistance \\
\hline
\end{tabular}

Table 2 Drug Sensitivity Test of $P$. aeruginosa

\begin{tabular}{|c|c|c|}
\hline \multicolumn{3}{|l|}{ P. aeruginosa ++++ } \\
\hline Antibiotics & MIC (ug/mL) & Results \\
\hline Amikacin & $\leq 8$ & Sensitive \\
\hline Aztreonam & 16 & Intermediary \\
\hline Cefepime & 8 & Sensitive \\
\hline Ceftazidime & 16 & Intermediary \\
\hline Ciprofloxacin & $>2$ & Resistance \\
\hline Polymyxin & $\leq 0.5$ & \\
\hline Gentamicin & $\leq 2$ & Sensitive \\
\hline Imipenem & $>8$ & Resistance \\
\hline Levofloxacin & $>8$ & Resistance \\
\hline Meropenem & 8 & Resistance \\
\hline Piperacillin & $>64$ & Resistance \\
\hline Piperacillin/ Tazobactam & $16 / 4$ & Sensitive \\
\hline
\end{tabular}

demonstrated the erythrocyte sedimentation rate (ESR), CRP and procalcitonin were negative; AST 14U/L, total protein $63.2 \mathrm{~g} / \mathrm{L}$, albumin $30.3 \mathrm{~g} / \mathrm{L}$, total calcium $2.1 \mathrm{mmol} /$ $\mathrm{L}$, phosphorus $0.84 \mathrm{mmol} / \mathrm{L}$, sialic acid $723 \mathrm{mg} / \mathrm{L}$. On day 23 , chest CT was rechecked to confirm the curative effect, which showed the formation of a pulmonary thick-walled cavity in the apical segment of the left upper lobe, with multiple small opacities, branching signs, bronchial wall thickening, and fibrous lesion. The inflammation of the cavity wall was obviously reduced compared with the first CT images (Figure 2). The patient was discharged in a stable condition on day 35 , and he was given acetylcysteine tablets $0.6 \mathrm{~g} / \mathrm{box}, 1$ tablet, bid. The final diagnoses on discharge were represented: 1 . Severe pneumonia 2. Sepsis
3. Septic shock 4. MODS 5. Type II respiratory failure 6 . Heart failure 7. Acute renal insufficiency 8. Metabolic acidosis 9. Chronic bronchitis 10. Hypoproteinemia.

After he was discharged, a regular follow-up of 2 weeks was performed to monitor his condition until most indicators came back to the normal level. The results of laboratory examinations showed as follows (September 17, 2019): Blood routine: white blood cell $9.57 \times 10^{\wedge} 9 / \mathrm{L}$, neutrophil count $6.78 \times 10^{\wedge} 9 / \mathrm{L}$, lymphocyte count $1.24 \times 10^{\wedge} 9 / \mathrm{L}$, neutrophil percentage $70.8 \%$, lymphocyte percentage $13 \%$, red blood cell count $3.92 \times 10^{\wedge} 12 / \mathrm{L}$, hemoglobin $111 \mathrm{~g} / \mathrm{L}$, hematocrit $0.35 \mathrm{~L} / \mathrm{L}$, mean corpuscular hemoglobin $318 \mathrm{~g} / \mathrm{L}$, platelet distribution width $9.2 \mathrm{fl}$, C-reactive protein (CRP) $19.04 \mathrm{mg} / \mathrm{L}$, PCT $0.19 \mathrm{ng} / \mathrm{mL}$; hepatorenal function: ALT 25U/L, AST 37U/L, Creatinine $75 \mathrm{umol} / \mathrm{L}$. As shown above, most laboratory results were improved, and the physical examination was normal.

\section{Discussion}

$P$. aeruginosa has strong virulence, producing various toxins, including exotoxins and enzymes, it is common to occur in people with immunocompromised or structural lung diseases, such as chronic obstructive pulmonary disease, bronchiectasis and cystic fibrosis. P. aeruginosa pneumonia accounts for up to $18 \%$ of nosocomial pneumonia cases, making it one of the most frequently isolated pathogens. ${ }^{8}$ Once the $P$. aeruginosa infection established, this pathogen in the $\mathrm{CF}$ airways develops into chronic infections. Ultimately, $60 \%$ to $80 \%$ of adults will become chronically infected with $P$. aeruginosa. ${ }^{9}$ Most of these cases shared some similar features, including rapid progression and severe complications. ${ }^{4,6,7}$

In this case, a 67-year-old male with no previous systemic disease. The CT after admission showed the dense consolidation site in the right lung, accompanying by mediastinal and right hilar lymphadenopathy. Sputum sample results showed $P$. aeruginosa and multidrugresistant Acinetobacter baumannii, multi-infection largely increased the therapeutic difficulty and the diagnosis of severe community-acquired pneumonia ( $P$. aeruginosa) can be confirmed. The male to female ratio of PCAP patients is $1.15: 1$, with a median onset age of (45 \pm 19$)$ years old. ${ }^{4}$ The common manifestations of PCAP are fever, cough and chest pain, usually involving the right upper lobe. Comparing to other types of CAP, PCAP patients are inclined to have respiratory failure and septic shock. ${ }^{10}$ 

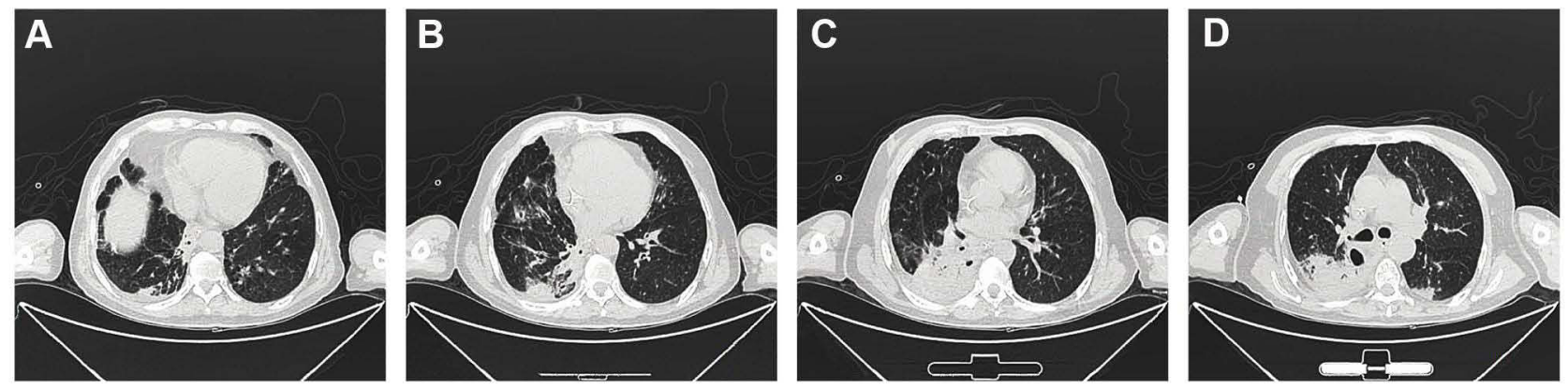

Figure 2 Computerized tomography was carried out on Day 23 after hospitalization. The dense consolidation and the inflammation of the cavity wall were reduced comparing with the first CT images.

$\mathrm{CT}$ is an efficient tool for picking up lung pathologies with high resolution, which could easily and accurately differentiate similar lung diseases, especially among critically ill patients. Comparing with conventional chest X-ray, CT can reveal small mild infiltrates which easily be neglected. ${ }^{11}$ However, some lung pathologies have similar appearances on CT scan, it is difficult to establish the differential diagnosis in patients with various lung diseases, such as ARDS and pneumonia. Hence, pathogen culture and relevant drug-sensitive test should be performed as a supplement way to guide the diagnosis decision and therapeutic strategies. Conventional specimen for culture is collected from the patient's sputum, while intubated patients rely on endotracheal aspirates (ETA). ${ }^{12}$ In recent years, the approaches of protected specimen brush or bronchoalveolar lavage (BAL) are also widely applied. Both the pathogen culture and drug-sensitive test require approximately $48 \mathrm{~h}$ to $72 \mathrm{~h}$ from sample acquisition to result delivery. ${ }^{13}$ Before the examination results of pathogen culture, empirical antibiotic treatment with administration of broad-spectrum antibiotics to cover the potential pathogens is needed. ${ }^{14}$

The prognosis of PCAP patients is firmly related to the effective anti- $P$. aeruginosa antibiotic therapy within 48 hours after admission. ${ }^{15}$ Sibila et al reported that the 30 day mortality rate would increase without effective antibiotic therapy in the first 48 hours. ${ }^{16}$ In the present case, the patient was initially provided with imipenem and cilastatin sodium $1.0 \mathrm{~g} \mathrm{q} 12 \mathrm{~h}$, linezolid $0.6 \mathrm{~g} \mathrm{q} 12 \mathrm{~h}$, and moxifloxacin $0.4 \mathrm{~g} \mathrm{qd}$. After a 3-day treatment, his symptoms were not alleviated with the maximum body temperature of $38.5^{\circ} \mathrm{C}$. Then we changed his treatment to the combination of polymyxin B and amikacin, instead of imipenem and cilastatin sodium, based on the sputum cultures. And the P. aeruginosa infection was under control.
Although the route of infection for $P$. aeruginosa CAP remains unknown, few previous studies have mentioned the related risk factors for PCAP. Contaminated aerosols have been referred that PCAP patients have used household humidifiers and hydrotherapy equipment, and isolated P. aeruginosa from the suspected contaminated water sample has been detected. ${ }^{4}$ Besides, workers engaged in metal casting and welding may get infected from inhaling dust contaminated by P. aeruginosa.$^{17}$ It is referred that the PCAP outbreak is relevant with the limited water consumption during droughts and water shortages, this could lead to the increased water pressure in the municipal water supply system, promoting the growth of $P$. aeruginosa biofilms. ${ }^{18}$ In the current case, this patient is a farmer, without any suspicious history of high-risk occupational exposure. However, he was caught in the rain and then the symptoms appeared, and he has a long smoking history. The possible reason might be that getting wet in the rain weakened his immunity, which might be the main reason for him to get infected by P. aeruginosa.

Most of the PCAP location involved the right upper lobe, approximately accounting for $2 / 3$ of patients. Interestingly, in line with previous studies, ${ }^{4}$ our patient also showed a dense consolidation site in the right lung, accompanying with mediastinal and right hilar lymphadenopathy by CT examination. The explanation of this phenomenon remains elusive. P. aeruginosa is an aerobic bacterium, we hypothesize that the upper lobes provide a more favorable environment under higher ventilation to perfusion ratios. Hence, we should consider P. aeruginosa in the differential diagnosis when we meet patients with distinctly upper lobe pneumonia. A low index of suspicion can be beneficial as chest X-ray may lag behind clinical manifestations, and necrotizing pneumonia at an early course can appear as consolidation only, leading to an underestimation of the degree of parenchymal destruction. ${ }^{19}$ 


\section{Conclusion}

Altogether, $P$. aeruginosa is an uncommon but fatal causative pathogen for CAP, we need to keep close attention due to its high mortality. It is crucial to perform CT and microbe culture to make the confirmative diagnosis, and empirical antibiotic treatment is needed to be initiated as soon as possible. PCAP is still rarely reported at present, further studies are needed to identify the infectious source, effective examination, and optimal therapy including medications and duration.

\section{Ethics Approval and Consent to Participate}

The patient in our case has signed the informed consent.

\section{Consent for Publication}

This study has been reviewed and approved by the Research Ethics Committee of the Central Hospital of Qingdao (KY-P202000301). The patient provided informed consent for publication of the clinical details including lung CT images, and written informed consent was obtained.

\section{Acknowledgments}

The authors would like to thank all study participants who were enrolled in this study.

\section{Funding}

There is no funding to report.

\section{Disclosure}

The authors declare that they have no competing interests.

\section{References}

1. Rello J, Borgatta B, Lisboa T. Risk factors for Pseudomonas aeruginosa pneumonia in the early twenty-first century. Intensive Care Med. 2013;39(12):2204-2206. doi:10.1007/s00134-013-3046-1

2. Cilloniz C, Ewig S, Polverino E, et al. Microbial aetiology of community-acquired pneumonia and its relation to severity. Thorax. 2011;66(4):340-346. doi:10.1136/thx.2010.143982

3. Sakamoto N, Tsuchiya K, Hikone M. Community-acquired necrotizing pneumonia with bacteremia caused by Pseudomonas aeruginosa in a patient with emphysema: an autopsy case report. Respir Investig. 2018;56:189-194.

4. Maharaj S, Isache C, Seegobin K, et al. Necrotizing Pseudomonas aeruginosa community-acquired pneumonia: a case report and review of the literature. Case Rep Infect Dis. 2017;2017:1717492. doi:10.1155/2017/1717492
5. Hatchette TF, Gupta R, Marrie TJ. Pseudomonas aeruginosa community-acquired pneumonia in previously healthy adults: case report and review of the literature. Clin Infect Dis. 2000;31 (6):1349-1356. doi:10.1086/317486

6. Sakamoto N, Tsuchiya K, Hikone M. Community-acquired necrotizing pneumonia with bacteremia caused by Pseudomonas aeruginosa in a patient with emphysema: an autopsy case report. Respir Investig. 2018;56(2):189-194. doi:10.1016/j.resinv.2017.12.008

7. Riviere P, Patin D, Delaporte E, et al. Septic shock secondary to an acute necrotizing community-acquired pneumonia with bacteremia due to Pseudomonas aeruginosa. IDCases. 2019;17:e00563. doi:10.1016/j.idcr.2019.e00563

8. Weiner LM, Webb AK, Limbago B, et al. Antimicrobial-resistant pathogens associated with healthcare-associated infections: summary of data reported to the national healthcare safety network at the centers for disease control and prevention, 2011-2014. Infect Control Hosp Epidemiol. 2016;37(11):1288-1301. doi:10.1017/ ice. 2016.174

9. Hauser AR, Jain M, Bar-Meir M, et al. Clinical significance of microbial infection and adaptation in cystic fibrosis. Clin Microbiol Rev. 2011;24(1):29-70.

10. Takajo D, Iwaya K, Katsurada Y, et al. Community-acquired lobar pneumonia caused by Pseudomonas aeruginosa infection in Japan: a case report with histological and immunohistochemical examination. Pathol Int. 2014;64(5):224-230. doi:10.1111/pin.12162

11. Xu E, Pérez-Torres D, Fragkou PC, et al. Nosocomial pneumonia in the era of multidrug-resistance: updates in diagnosis and management. Microorganisms. 2021;9(3):534. doi:10.3390/ microorganisms 9030534

12. Kalil AC, Metersky ML, Klompas M, et al. Executive summary: management of adults with hospital-acquired and ventilator-associated pneumonia: 2016 clinical practice guidelines by the infectious diseases society of America and the American thoracic society. Clin Infect Dis. 2016;63(5):575-582. doi:10.1093/ cid/ciw504

13. Yoo IY, Huh K, Shim HJ, et al. Evaluation of the BioFire FilmArray pneumonia panel for rapid detection of respiratory bacterial pathogens and antibiotic resistance genes in sputum and endotracheal aspirate specimens. Int J Infect Dis. 2020;95:326-331. doi:10.1016/ j.ijid.2020.03.024

14. Prina E, Ranzani OT, Torres A. Community-acquired pneumonia. Lancet. 2015;386(9998):1097-1108. doi:10.1016/S0140-6736(15) 60733-4

15. Garcia-Vidal C, Fernandez-Sabe N, Carratala J, et al. Early mortality in patients with community-acquired pneumonia: causes and risk factors. Eur Repirat J. 2008;32(3):733-739. doi:10.1183/ 09031936.00128107

16. Sibila O, Laserna E, Maselli DJ, et al. Risk factors and antibiotic therapy in P. aeruginosa community-acquired pneumonia. Respirology. 2015;20(4):660-666. doi:10.1111/resp.12506

17. Serota DP, Sexton ME, Kraft CS, et al. Severe community-acquired pneumonia due to Acinetobacter baumannii in North America: case report and review of the literature. Open Forum Infect Dis. 2018;5(3): ofy044. doi:10.1093/ofid/ofy044

18. John T-J, Lalla U, Taljaard JJ, Slabbert J, Koegelenberg CFN. An outbreak of community-acquired Pseudomonas aeruginosa pneumonia in a setting of high water stress. QJM. 2017:855-856. doi:10.1093/qjmed/hcx148

19. Tsai YF, Ku YH. Necrotizing pneumonia: a rare complication of pneumonia requiring special consideration. Curr Opin Pulm Med. 2012;18(3):246-252. doi:10.1097/MCP.0b013e3283521022 


\section{Publish your work in this journal}

Infection and Drug Resistance is an international, peer-reviewed openaccess journal that focuses on the optimal treatment of infection (bacterial, fungal and viral) and the development and institution of preventive strategies to minimize the development and spread of resistance. The journal is specifically concerned with the epidemiology of antibiotic resistance and the mechanisms of resistance development and diffusion in both hospitals and the community. The manuscript management system is completely online and includes a very quick and fair peerreview system, which is all easy to use. Visit http://www.dovepress.com/ testimonials.php to read real quotes from published authors. 